\title{
Thin Interface Asymptotics for an Energy/Entropy Approach to Phase-Field Models with Unequal Conductivities
}

\section{G. B. McFadden}

U.S. DEPARTMENT OF COMMERCE

Technology Administration

National Institute of Standards

and Technology

100 Bureau Drive Stop 8910

Gaithersburg, MD 20899-8910

\section{A. A. Wheeler}

Faculty of Mathematical Studies

University of Southampton

Southampton SO17 1EJ, UK

\section{M. Anderson}

Department of Mathematics George Mason University

Fairfax, VA 22030

QC 



\section{Thin Interface Asymptotics for an Energy/Entropy Approach to Phase-Field Models with Unequal Conductivities}

\section{G. B. McFadden}

U.S. DEPARTMENT OF COMMERCE Technology Administration

National Institute of Standards and Technology

100 Bureau Drive Stop 8910

Gaithersburg, MD 20899-8910

\section{A. A. Wheeler}

Faculty of Mathematical Studies University of Southampton Southampton S017 1BJ, UK

\section{M. Anderson}

Department of Mathematics George Mason University Fairfax, VA 22030

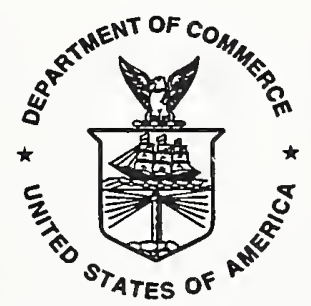

U.S. DEPARTMENT OF COMMERCE William M. Daley, Secretary

TECHNOLOGY ADMINISTRATION Gary R. Bachula, Acting Under Secretary for Technology

NATIONAL INSTITUTE OF STANDARDS AND TECHNOLOGY

Raymond G. Kammer, Director 



\title{
Thin Interface Asymptotics for an Energy/Entropy Approach to Phase-Field Models with Unequal Conductivities
}

\author{
G. B. McFadden \\ National Institute of Standards and Technology \\ Gaithersburg, MD 20899-8910, USA
}

\author{
A. A. Wheeler \\ Faculty of Mathematical Studies \\ University of Southampton \\ Southampton SO17 1BJ, UK \\ D. M. Anderson \\ Department of Mathematics \\ George Mason University \\ Fairfax, VA 22030
}

\begin{abstract}
Karma and Rappel [1] recently developed a new sharp interface asymptotic analysis of the phase-field equations that is especially appropriate for modeling dendritic growth at low undercoolings. Their approach relieves a stringent restriction on the interface thickness that applies in the conventional asymptotic analysis, and has the added advantage that interfacial kinetic effects can also be eliminated. However, their analysis focussed on the case of equal thermal conductivities in the solid and liquid phases; when applied to a standard phase-field model with unequal conductivities, anomalous terms arise in the limiting forms of the boundary conditions for the interfacial temperature that are not present in conventional sharp-interface solidification models, as discussed further by Almgren [2]. In this paper we apply their asymptotic methodology to a generalized phase-field model which is derived using a thermodynamically consistent approach that is based on independent entropy and internal energy gradient functionals that include double wells in both the entropy and internal energy densities. The
\end{abstract}


additional degrees of freedom associated with the generalized phase-field equations can be chosen to eliminate the anomalous terms that arise for unequal conductivities.

PACS: 81.10.Aj, 64.70.Dv, 66.10.Cb, 81.10.Mx

Keywords: phase-field model, sharp interface analysis, thin interface analysis, dendritic growth, isothermal asymptotics.

\section{Introduction}

Various asymptotic limits can be used to relate diffuse interface models of solidification to conventional free boundary problems that involve sharp interfaces of zero width (see, for example, [3]). In particular, the size of the diffuse interface width $\ell$ relative to other characteristic length scales in the problem determines the specific form of the free boundary problem that is obtained as $\ell$ becomes small. In this paper we will be especially concerned with the effects of capillarity and interface attachment kinetics, as described by the modified Gibbs-Thomson boundary condition,

$$
\frac{T-T_{M}}{L_{V} / c_{P}}=-\ell_{c} \mathcal{H}-V / V_{0}
$$

which relates the interface mean curvature $\mathcal{H}$, and normal velocity $V$, to the dimensionless interface temperature, $u=\left(T-T_{M}\right) /\left[L_{V} / c_{P}\right]$, where $T$ is the temperature, $T_{M}$ is the melting point, $L_{V}$ is the latent heat per unit volume, and $c_{P}$ is the heat capacity per unit volume. Here $\ell_{c}=T_{M} \gamma /\left[L_{V}^{2} / c_{P}\right]$ is a capillary length based on the surface free energy $\gamma$, and $V_{0}=\mu\left[L_{V} / c_{P}\right]$ is a characteristic velocity based on the kinetic attachment coefficient, $\mu$. For discussion purposes we consider the growth of a dendrite with tip radius $R$ into an undercooled melt [4]; in more general circumstances, $R$ might be representative of a typical macroscopic length scale such as a container size. For dendritic solidification at large undercoolings the growth is rapid and the radius of curvature of the tip is relatively small, so that the effects of capillarity and kinetics on the interface temperature in Eq. (1) can be significant. In this regime, sharp interface limits of the phase field equations have been performed [5-10] that assume the interface temperature $u$ is of order unity in the small parameter $\ell / R$, which allows 
the contributions from capillarity and kinetics to be of order unity as well. In this limit $\ell$ is also assumed to be small compared to $\ell_{c}$, which presents a stringent resolution requirement for a numerical computation that aspires to describe this limiting case. At low undercoolings, on the other hand, dendrites grow more slowly and have a larger radius of curvature, so it is reasonable to model the effects of capillarity and kinetics as small corrections. Karma and Rappel [1] refer to the corresponding analysis as the "thin interface limit," wherein one assumes $\ell \ll R$ but allows $\ell \sim \ell_{c}$. Almgren [2] has described this analysis as "isothermal asymptotics," since to leading order in $\ell / R$ the temperature is isothermal throughout the interfacial region, with $u=O(\ell / R)$. A technical difficulty with this analysis is that for unequal thermal conductivities in the liquid and solid, the thin interface limit can lead to a discontinuity in the temperature field across the interface as well as the appearance of an anomalous term proportional to the interfacial temperature gradients in the modified Gibbs-Thomson equation. Almgren shows that a continuous temperature field and a GibbsThomson equation independent of the interfacial temperature gradients can be achieved by appropriate alterations of the interpolation functions that are used to represent the bulk thermophysical quantities across the diffuse interface.

The standard phase-field model, a version of the Model $\mathrm{H}$ of Halperin, Hohenberg, and Ma [11], was originally derived on the basis of a gradient functional for the free energy of the system, with dynamics that ensure that the free energy decreases with time [12-16]. The time rate of change of the order parameter is coupled to the energy equation to incorporate the release of latent heat as solidification occurs. Penrose and Fife [17] (see also [18]) derived the phase-field equations on the basis of positive entropy production for a system that is based on a gradient functional for the entropy of the system. Here we consider a generalized phase field model that is based on gradient functionals for both the entropy and internal energy of the system; such models have been considered by a number of authors [19-23]. The generalized model contains additional degrees of freedom that we use here to eliminate the anomalous terms that arise in the asymptotic analysis of Karma and Rappel in the case of unequal thermal conductivities. 
The kinetic attachment coefficient $\mu$ that appears in the characteristic velocity $V_{0}$ in Eq. (1) has been measured for relatively few materials. For this reason, and because the effect of kinetics is usually expected to be small at low to moderate rates of solidification for metals and other molecularly-simple materials, many sharp interface models ignore the effect of kinetics by setting $1 / \mu=0$. Karma and Rappel show how their approach allows the effects of kinetics to be eliminated in the standard phase-field model by an appropriate choice of the parameters in the model. We also address this issue for the generalized phase field model, and find that the effects of kinetics can be eliminated for a significant range of the ratio of thermal conductivities as well.

In the next section we describe the phase field models that we consider. Asymptotic analyses of these models are presented in Section 3, followed by discussion and concluding sections.

\section{Phase-Field Model}

A common version of the standard phase-field equations for a pure material can be written in the form

$$
\begin{gathered}
\frac{1}{M} \phi_{t}=K \nabla^{2} \phi-\frac{W}{2} g^{\prime}(\phi)-L_{V} \frac{\left(T-T_{M}\right)}{T_{M}} p^{\prime}(\phi), \\
c_{P} T_{t}-L_{V} r^{\prime}(\phi) \phi_{t}=\nabla \cdot[k(\phi) \nabla T],
\end{gathered}
$$

where the phase field $\phi(\vec{x}, t)$ is a nonconserved order parameter that labels the phases, with $\phi=1$ in the solid phase and $\phi=0$ in the liquid phase. Here $M>0$ is a mobility coefficient, $K>0$ is a gradient energy coefficient, $W>0$ is a barrier height for the double well potential function $g(\phi)=\phi^{2}(1-\phi)^{2}$ and $p(\phi)=\phi^{2}(3-2 \phi)$. The function $k(\phi)$ permits different thermal conductivities $k_{L}=k(0)$ and $k_{S}=k(1)$ in the bulk liquid and solid phases, respectively; specific forms for $k(\phi)$ will be discussed below. The function $r(\phi)$ is assumed to have $r(0)=0$ and $r(1)=1$. Examples include the cases $r(\phi)=p(\phi)$ and $r(\phi)=\phi$. Following Almgren we refer to the system with $r(\phi)=p(\phi)$ and $r(\phi)=\phi$ as the gradient 
equations and the nongradient equations, respectively; the reason for this terminology lies in how the equations are derived (see [2] and below).

An isothermal version of this model has an associated bulk free energy density of the form

$$
f(\phi, T)=\frac{W}{2} g(\phi)+\frac{L_{V}\left(T-T_{M}\right)}{T_{M}} p(\phi) .
$$

In this case the phase-field equation (2) admits a one-dimensional traveling wave of the form

$$
\phi(x, t)=\frac{1}{2}\left\{1-\tanh \left(\frac{x-V t}{2 \ell}\right)\right\},
$$

where the interface velocity is given by $V=6 M L_{V} \ell\left(T_{M}-T\right) / T_{M}$. The interface width $\ell$, surface energy $\gamma$, and kinetic coefficient $\mu$ that are associated with this solution are given by

$$
\ell=\sqrt{K / W}, \quad \gamma=\frac{\sqrt{K W}}{6}, \quad \mu=\frac{6 M L_{V} \ell}{T_{M}},
$$

where the latter expression follows from the definition $V=\mu\left(T_{M}-T\right)$. Karma and Rappel [1] provide an alternate interpretation of the kinetic effect through their thin interface limit, which is also discussed below; this limit is intrinsically non-isothermal, so that the above interpretation does not apply.

The governing equations (2) and (3) can be derived from an entropy functional following the formalism of irreversible thermodynamics $[17,18]$. A generalized version of these equations can be derived by using both an internal energy functional $\mathcal{E}$ and an entropy functional $\mathcal{S}$, which we write in the form [19-24]

$$
\begin{aligned}
& \mathcal{E}=\int_{V}\left\{e(\phi, T)+\frac{K_{E}}{2}|\nabla \phi|^{2}\right\} d V, \\
& \mathcal{S}=\int_{V}\left\{s(\phi, T)-\frac{K_{S}}{2}|\nabla \phi|^{2}\right\} d V,
\end{aligned}
$$

where $K_{E}$ and $K_{S}$ are gradient coefficients that will be assumed to be constant, and $e$ and $s$ are the internal energy and entropy densities, respectively. By starting with a free 
energy density $f(T, \phi)$, it is thermodynamically consistent to take $s(T, \phi)=-\partial f / \partial T$ and $e(T, \phi)=f(T, \phi)+T s(T, \phi)$.

An associated free energy functional $\mathcal{F}$ can be defined for the system, which is given by

$$
\mathcal{F}=\int_{V}\left\{f(\phi, T)+\frac{\left(K_{E}+T K_{S}\right)}{2}|\nabla \phi|^{2}\right\} d V
$$

Anisotropic versions of the model can be obtained by modification of the gradient terms $|\nabla \phi|^{2}$, as described in more detail below. For simplicity we will assume that the densities and specific heats are uniform throughout the system.

The derivation of the governing equations may be based on conservation laws for energy and entropy written in the form

$$
\begin{gathered}
\frac{d \mathcal{E}}{d t}+\int_{\delta V} \vec{J}_{E} \cdot \widehat{n} d A=0, \\
\frac{d \mathcal{S}}{d t}+\int_{\delta V} \vec{J}_{S} \cdot \widehat{n} d A=\int_{V} \dot{s}_{P} d V
\end{gathered}
$$

where $\vec{J}_{E}$ and $\vec{J}_{S}$ represent the flux of energy and entropy through the boundary $\delta V$, which has outward unit normal $\widehat{n}$, and $\dot{s}_{P}$ represents the entropy production term. Governing equations that guarantee positive entropy production can be obtained by the choice

$$
\begin{aligned}
& \vec{J}_{E}=-k \nabla T-K_{E} \phi_{t} \nabla \phi, \\
& \vec{J}_{S}=-\frac{k}{T} \nabla T+K_{S} \phi_{t} \nabla \phi,
\end{aligned}
$$

where the terms proportional to the gradient coefficients represent non-classical fluxes associated with the interfacial region [18]. The entropy production is then given by

$$
T \dot{s}_{P}=\frac{k}{T}|\nabla T|^{2}+\phi_{t}\left[\left(K_{E}+T K_{S}\right) \nabla^{2} \phi-f_{\phi}\right]
$$

and the corresponding phase-field equation [cf. Eq. (17)] is chosen so that the latter expres- 
sion takes the form $k|\nabla T|^{2} / T+\phi_{t}^{2} / M$, with $M>0$.

In this paper we consider a free energy of the form

$$
f(\phi, T)=\frac{\left(W_{E}+T W_{S}\right)}{2} g(\phi)+\frac{L_{V}\left(T-T_{M}\right)}{T_{M}} p(\phi)-c_{P}\left(T \ln \left[T / T_{M}\right]-T\right) .
$$

The corresponding internal entropy and energy densities may be found, using the relations $s=-\partial f / \partial T$ and $e=f+T s$, to be

$$
e(\phi, T)=c_{P} T+\frac{W_{E}}{2} g(\phi)-L_{V} p(\phi), \quad s(\phi, T)=c_{P} \ln \left[T / T_{m}\right]-\frac{W_{S}}{2} g(\phi)-\frac{L_{V}}{T_{M}} p(\phi) .
$$

Here $W_{E}$ and $W_{S}$ are constant double well heights for internal energy and entropy, respectively. The phase-field models considered in Refs. [17] and [18] correspond to a specific entropic version formulation obtained from our model when $K_{E}=W_{E}=0$.

The generalized phase-field equations corresponding to this model take the form

$$
\begin{gathered}
\frac{1}{M} \phi_{t}=\left(K_{E}+T K_{S}\right) \nabla^{2} \phi-\frac{\left(W_{E}+T W_{S}\right)}{2} g^{\prime}(\phi)-\frac{L_{V}\left(T-T_{M}\right)}{T_{M}} p^{\prime}(\phi), \\
c_{P} T_{t}-L_{V} p^{\prime}(\phi) \phi_{t}+\frac{W_{E}}{2} g^{\prime}(\phi) \phi_{t}=\nabla \cdot[k(\phi) \nabla T]+K_{E} \phi_{t} \nabla^{2} \phi .
\end{gathered}
$$

This generalized model includes two additional temperature-dependent terms $\left(T-T_{M}\right) K_{S} \nabla^{2} \phi$ and $\left(T-T_{M}\right) W_{S} g^{\prime}(\phi) / 2$ in the phase-field equation, as well as two additional terms $W_{E} \phi_{t} g^{\prime}(\phi) / 2$ and $K_{E} \phi_{t} \nabla^{2} \phi$ in the energy equation, when compared to the standard version (2) and (3). If these terms are omitted, these equations are similar to the standard version, but with $p^{\prime}(\phi)$ appearing in place of $r^{\prime}(\phi)$ in the energy equation. We will refer to equations (17) and (18) with $p(\phi)$ replaced by $r(\phi)$ in (18) as the generalized phase-field equations. The forms of the generalized phase-field equations corresponding to the particular cases $r(\phi)=p(\phi)$ and $r(\phi)=\phi$ will be termed the generalized gradient equations and the generalized nongradient equations, respectively.

The question of whether $K_{E}, K_{S}, W_{E}$ and $W_{S}$ represent measurable physical quantities is an important issue that concerns the physical validity of Eqs. (17) and (18). Here we 
adopt a pragmatic approach, and view the formal derivation of these equations as a means of suggesting modified forms of the governing equations that can be advantageous from the viewpoint of asymptotics and computations. For example, Karma and Rappel [1] note that there are computational advantages to using nongradient governing equations that do not result from a formal thermodynamic derivation; they suggest the use of the function $r(\phi)=\phi$ rather than $r(\phi)=p(\phi)$ in the latent heat term of the energy equation. In a similar vein, Almgren considers the question of choosing the specific form of the interpolation functions $p(\phi), r(\phi)$, and $k(\phi)$ to eliminate unwanted terms in the thin interface asymptotics that arise when $k_{L} \neq k_{S}$. Here we examine the effect on the thin interface asymptotics of introducing the terms involving $K_{E}, K_{S}, W_{E}$ and $W_{S}$ for fixed forms for the interpolation functions.

\subsection{Dimensionless Equations}

We make the governing equations (17) and (18) dimensionless by using the macroscopic length scale $R$, the thermal time scale $R^{2} / \bar{\kappa}$, and the temperature scale $L_{V} / c_{P}$; here $\bar{\kappa}=$ $\left(\kappa_{L}+\kappa_{L}\right) / 2$ is the average of the thermal diffusivities $\kappa_{L}=k_{L} / c_{P}$ and $\kappa_{S}=k_{S} / c_{P}$ in the liquid and solid, respectively. We retain the original definitions of the interface width $\ell$ and surface energy $\gamma$ by using $K=\left(K_{E}+T_{M} K_{S}\right)$ and $W=\left(W_{E}+T_{M} W_{S}\right)$ in Eq. (6). The resulting dimensionless equations can be written in the form

$$
\begin{gathered}
\epsilon^{2} \tau \phi_{t}=\epsilon^{2}(1+\alpha u) \nabla^{2} \phi-\frac{(1+\beta u)}{2} g^{\prime}(\phi)-\lambda u p^{\prime}(\phi), \\
u_{t}-r^{\prime}(\phi) \phi_{t}+\frac{\delta}{2} g^{\prime}(\phi) \phi_{t}=\nabla \cdot[Q(\phi) \nabla u]+\epsilon^{2} \nu \phi_{t} \nabla^{2} \phi,
\end{gathered}
$$

where we have defined the conventional dimensionless parameters

$$
\epsilon=\frac{\ell}{R}, \quad \lambda=\frac{\ell}{6 \ell_{c}}, \quad \tau=\frac{\bar{\kappa} T_{m} c_{P}}{6 \ell \ell_{c} M L_{V}^{2}},
$$


that usually appear in the phase-field equations, as well as the parameters

$$
\alpha=\frac{K_{S}\left[L_{V} / c_{P}\right]}{K}, \quad \beta=\frac{W_{S}\left[L_{V} / c_{P}\right]}{W}, \quad \delta=\frac{W_{E}}{L_{V}}, \quad \nu=\frac{K_{E}}{L_{V} \ell^{2}},
$$

that appear in the generalized model. For now we will treat $\alpha, \beta, \delta$, and $\nu$ as independent parameters, and will return to the question of the relationship between these parameters below. We have also defined the dimensionless function $Q(\phi)=k(\phi) /\left[\bar{\kappa} c_{P}\right]$ with $Q(0)=$ $Q_{L}=\kappa_{L} / \bar{\kappa}$ and $Q(1)=Q_{S}=\kappa_{S} / \bar{\kappa}$. We note that since $Q_{L}$ and $Q_{S}$ are normalized in terms of their mean value, we have $Q_{L}+Q_{S}=2$ and hence $0<Q_{L}<2$ and $0<Q_{S}<2$.

The relative sizes of the dimensionless parameters determine what type of sharp interface analysis is appropriate in deriving the limiting form of the associated free boundary problem. The original approach to the sharp interface analysis, which we will refer to as the "classical analysis," and the thin interface analysis of Karma and Rappel differ particularly in the assumptions concerning the scaling of the factor $\lambda u$ in Eq. (19).

\section{$3 \quad$ Asymptotic Analyses}

The various sharp interface limits use the method of matched asymptotic expansions, whereby an outer solution, valid away from the interface, is matched to an inner solution that is valid in the interfacial region [6]; for brevity we only sketch the procedure. The inner expansion near a point $\vec{x}_{0}$ on the interface is described via a stretched coordinate $\zeta$, with $\vec{x}=\vec{x}_{0}+\epsilon \zeta \widehat{n}$, where $\widehat{n}$ is the local unit normal to the interface. The low order terms in an inner expansion of the solution can be obtained by using the relations

$$
\nabla^{2}=\frac{1}{\epsilon^{2}} \partial_{\zeta}^{2}+\frac{\widetilde{\mathcal{H}}}{\epsilon} \partial_{\zeta}+O(1), \quad \partial_{t}=\frac{-v_{n}}{\epsilon} \partial_{\zeta}+O(1)
$$

where the dimensionless mean curvature $\widetilde{\mathcal{H}}=R \mathcal{H}$ and the dimensionless interface speed $v_{n}=V R / \bar{\kappa}$ are assumed to be of order unity. In the inner region the temperature and phase 
field are expanded in powers of $\epsilon$,

$$
\begin{aligned}
& \phi=\phi^{(0)}+\epsilon \phi^{(1)}+O\left(\epsilon^{2}\right), \\
& u=u^{(0)}+\epsilon u^{(1)}+O\left(\epsilon^{2}\right),
\end{aligned}
$$

and the resulting equations are solved term by term in $\epsilon$, with far-field boundary conditions that are obtained by matching to the outer solution. The outer solution has $\phi(\vec{x})$ equal to zero or one to all orders in the liquid or solid respectively, and the thermal field has the expansion $U(\vec{x})=U^{(0)}(\vec{x})+\epsilon U^{(1)}(\vec{x})+O\left(\epsilon^{2}\right)$. The limiting behavior near a point $\vec{x}_{0}$ on the interface is given by

$$
U\left(\vec{x}_{0}+\epsilon \zeta \widehat{n}\right)=U_{ \pm}^{(0)}\left(\vec{x}_{0}\right)+\epsilon\left[U_{ \pm}^{(1)}\left(\vec{x}_{0}\right)+\zeta \frac{\partial U_{ \pm}^{(0)}}{\partial n}\left(\vec{x}_{0}\right)\right]+O\left(\epsilon^{2}\right)
$$

where $U_{+}^{(0)}$ and $U_{-}^{(0)}$ denote the limits of $U$ as $\zeta \rightarrow 0_{+}$and $\zeta \rightarrow 0_{-}$, respectively, as so forth. The inner solution must match with this behavior as $\zeta \rightarrow \pm \infty[6]$.

\subsection{Classical Analysis}

In this setting a standard model given by (19) and (20) with $\alpha, \beta, \delta$, and $\nu$ all set to zero is employed. The asymptotic analysis with $\ell<<\ell_{c}$ assumes that $\lambda=\ell /\left(6 \ell_{c}\right)$ is a small parameter, which allows one to take $u$ to be of order unity while relegating to $O(\epsilon)$ the term $\lambda u$. If we write $\lambda=\Lambda \epsilon$, then the leading order phase-field equation is given by

$$
\phi_{\zeta \zeta}^{(0)}-\frac{1}{2} g^{\prime}\left(\phi^{(0)}\right)=0
$$

with the solution

$$
\phi^{(0)}(\zeta)=\frac{1}{2}\left[1-\tanh \left(\frac{\zeta}{2}\right)\right]
$$


The leading order temperature equation is

$$
\left(Q\left(\phi^{(0)}\right) u_{\zeta}^{(0)}\right)_{\zeta}=0
$$

Integrating and matching with the outer solution twice in succession gives that $u^{(0)}=$ $U_{+}^{(0)}\left(\vec{x}_{0}\right)=U_{-}^{(0)}\left(\vec{x}_{0}\right)$ is constant.

The first order phase field equation is then

$$
\phi_{\zeta \zeta}^{(1)}-\frac{1}{2} g^{\prime \prime}\left(\phi^{(0)}\right) \phi^{(1)}=-\left(\tau v_{n}+\widetilde{\mathcal{H}}\right) \phi_{\zeta}^{(0)}+\Lambda u^{(0)} p^{\prime}\left(\phi^{(0)}\right),
$$

and a solvability condition for this equation then gives (c.f. Eq. (38) below)

$$
u^{(0)}=\frac{-\widetilde{\mathcal{H}}}{6 \Lambda}-\frac{\tau v_{n}}{6 \Lambda}=-\ell_{c} H-V / V_{0}
$$

which recovers the modified Gibbs-Thomson boundary condition (1), where the kinetic term agrees with the form of $V_{0}=\mu\left[L_{V} / c_{P}\right]$ given in Eqs. (6) and (21); thus both the isothermal traveling wave solution and the classical asymptotic analysis lead to the same definition of $\mu$ in terms of the phase field parameters.

The first order temperature equation is then

$$
v_{n} r^{\prime}\left(\phi^{(0)}\right) \phi_{\zeta}^{(0)}=\left(Q\left(\phi^{(0)}\right) u_{\zeta}^{(1)}\right)_{\zeta}
$$

By integrating this equation over the interval $-\infty<\zeta<\infty$ and using the matching conditions, the leading order heat flux boundary condition is recovered in the dimensionless form

$$
-v_{n}=Q_{L} G_{L}-Q_{S} G_{S}
$$

where $G_{L}=\partial U_{+}^{(0)} / \partial n$ and $G_{S}=\partial U_{-}^{(0)} / \partial n$ denote the local temperature gradients at the interface.

Almgren has considered the forms of the terms that occur at the next order in the classical 
analysis, and has discussed strategies for eliminating anomalous terms at that order so as to obtain higher-order accuracy in the Gibbs-Thomson equation [2]. Almgren also discusses the thin interface analysis of Karma and Rappel [1], to which we turn next.

\subsection{Thin Interface Analysis}

In the thin interface, or isothermal, analysis, $\lambda$ is assumed to be of order unity, but $\lambda u=$ $O(\epsilon)$ is still retained by taking $u^{(0)}=0$ so that $u=O(\epsilon)$. We will show that for the generalized phase-field equations the quantities $\alpha, \beta, \delta$ and $\nu$ may be chosen to ensure that both the interfacial temperature is continuous and the modified Gibbs-Thomson equation is independent of $G_{L}$ and $G_{S}$.

With the assumption that the parameters $\lambda, \tau, v_{n}, \widetilde{\mathcal{H}}, \alpha, \beta, \delta$ and $\nu$ are all of order unity, the leading and first order phase-field equations are then

$$
\begin{gathered}
\phi_{\zeta \zeta}^{(0)}-\frac{1}{2} g^{\prime}\left(\phi^{(0)}\right)=0, \\
\phi_{\zeta \zeta}^{(1)}-\frac{1}{2} g^{\prime \prime}\left(\phi^{(0)}\right) \phi^{(1)}=R_{1},
\end{gathered}
$$

where

$$
R_{1}=-\alpha u^{(1)} \phi_{\zeta \zeta}^{(0)}+\frac{\beta}{2} u^{(1)} g^{\prime}\left(\phi^{(0)}\right)-\left(\tau v_{n}+\widetilde{\mathcal{H}}\right) \phi_{\zeta}^{(0)}+\lambda u^{(1)} p^{\prime}\left(\phi^{(0)}\right)
$$

The leading order phase field solution is again given by Eq. (28). The first two terms of $R_{1}$ given by Eq. (36) can be simplified using Eq. (34) to give

$$
-\alpha u^{(1)} \phi_{\zeta \zeta}^{(0)}+\frac{\beta}{2} u^{(1)} g^{\prime}\left(\phi^{(0)}\right)=\frac{\widetilde{\beta}}{2} u^{(1)} g^{\prime}\left(\phi^{(0)}\right)
$$

where we have defined $\widetilde{\beta}=\beta-\alpha$. The solvability condition for Eq. (35) can then be written in the form

$$
0=\int_{-\infty}^{\infty} \phi_{\zeta}^{(0)} R_{1} d \zeta=\lambda \int_{-\infty}^{\infty} u^{(1)} p^{\prime}\left(\phi^{(0)}\right) \phi_{\zeta}^{(0)} d \zeta+\frac{\widetilde{\beta}}{2} \int_{-\infty}^{\infty} u^{(1)} g^{\prime}\left(\phi^{(0)}\right) \phi_{\zeta}^{(0)} d \zeta-\frac{1}{6}\left(\tau v_{n}+\widetilde{\mathcal{H}}\right)
$$


which involves the first order temperature solution $u^{(1)}$. We observe that only the combination $\beta-\alpha=\widetilde{\beta}$ enters the solvability condition.

With $u^{(0)}=0$ the temperature equation is satisfied identically to leading order. The first order temperature equation is

$$
v_{n} r^{\prime}\left(\phi^{(0)}\right) \phi_{\zeta}^{(0)}-\frac{\delta}{2} v_{n} g^{\prime}\left(\phi^{(0)}\right) \phi_{\zeta}^{(0)}=\left(Q\left(\phi^{(0)}\right) u_{\zeta}^{(1)}\right)_{\zeta}-\nu v_{n} \phi_{\zeta}^{(0)} \phi_{\zeta \zeta}^{(0)}
$$

which, when simplified by using Eq. (34) as above, may be integrated to yield

$$
Q\left(\phi^{(0)}\right) u_{\zeta}^{(1)}-v_{n} r\left(\phi^{(0)}\right)-\frac{\widetilde{\nu} v_{n}}{2}\left[\phi_{\zeta}^{(0)}\right]^{2}=Q_{L} G_{L}=Q_{S} G_{S}-v_{n}
$$

where we have defined $\widetilde{\nu}=\nu-\delta$. Here the constant of integration has been evaluated in two ways by taking $\zeta \rightarrow \pm \infty$ and applying the matching conditions (26). Note that only the combination $\nu-\delta=\widetilde{\nu}$ appears in this expression. The solution can then be expressed in the equivalent forms

$$
\begin{aligned}
u^{(1)}(\zeta) & =U_{+}^{(1)}\left(\vec{x}_{0}\right)+\zeta G_{L} \\
& -\int_{\zeta}^{\infty} \frac{1}{Q\left(\phi^{(0)}\right)}\left\{v_{n} r\left(\phi^{(0)}\right)+\frac{\widetilde{\nu} v_{n}}{2}\left[\phi_{\zeta}^{(0)}\right]^{2}-G_{L}\left[Q\left(\phi^{(0)}\right)-Q_{L}\right]\right\} d \eta \\
& =U_{-}^{(1)}\left(\vec{x}_{0}\right)+\zeta G_{S} \\
& +\int_{-\infty}^{\zeta} \frac{1}{Q\left(\phi^{(0)}\right)}\left\{v_{n}\left[r\left(\phi^{(0)}\right)-1\right]+\frac{\widetilde{\nu} v_{n}}{2}\left[\phi_{\zeta}^{(0)}\right]^{2}-G_{S}\left[Q\left(\phi^{(0)}\right)-Q_{S}\right]\right\} d \eta
\end{aligned}
$$

\subsubsection{Continuity of Temperature}

Subtracting the two equivalent forms for $u^{(1)}(0)$ provides an expression for the jump in the temperature across the interface,

$$
\begin{gathered}
U_{+}^{(1)}\left(\vec{x}_{0}\right)-U_{-}^{(1)}\left(\vec{x}_{0}\right)=\int_{0}^{\infty} \frac{1}{Q\left(\phi^{(0)}\right)}\left\{v_{n} r\left(\phi^{(0)}\right)+\frac{\widetilde{\nu} v_{n}}{2}\left[\phi_{\zeta}^{(0)}\right]^{2}-G_{L}\left[Q\left(\phi^{(0)}\right)-Q_{L}\right]\right\} d \eta \\
+\int_{-\infty}^{0} \frac{1}{Q\left(\phi^{(0)}\right)}\left\{v_{n}\left[r\left(\phi^{(0)}\right)-1\right]+\frac{\widetilde{\nu} v_{n}}{2}\left[\phi_{\zeta}^{(0)}\right]^{2}-G_{S}\left[Q\left(\phi^{(0)}\right)-Q_{S}\right]\right\} d \eta
\end{gathered}
$$


For $\tilde{\nu}=0$, Karma and Rappel [1] found that this expression vanishes for either $r(\phi)=$ $\phi$ or $r(\phi)=p(\phi)$ if the thermal properties in the liquid and solid phases are the same. Almgren [2] considered the more general case of unequal thermal properties, and noted that it is convenient to interpolate $1 / Q(\phi)$, since that quantity appears in the above integrand. Almgren discusses how to eliminate possible temperature discontinuities by choosing more elaborate interpolation functions for the case with $\widetilde{\nu}=0$. Here for $\widetilde{\nu} \neq 0$ we consider the simple choice

$$
\frac{1}{Q(\phi)}=\frac{r(\phi)}{Q_{S}}+\frac{[1-r(\phi)]}{Q_{L}} .
$$

where we interpolate using $r(\phi)$. For $r(\phi)=\phi$ the integrals in Eq. (43) can then be evaluated directly to yield

$$
U_{+}^{(1)}\left(\vec{x}_{0}\right)-U_{-}^{(1)}\left(\vec{x}_{0}\right)=v_{n}\left\{\frac{\tilde{\nu}}{24}\left(\frac{1}{Q_{L}}+\frac{1}{Q_{S}}\right)+\left(\frac{1}{Q_{L}}-\frac{1}{Q_{S}}\right)\right\}
$$

and for the choice $r(\phi)=p(\phi)=\phi^{2}(3-2 \phi)$ we similarly find

$$
U_{+}^{(1)}\left(\vec{x}_{0}\right)-U_{-}^{(1)}\left(\vec{x}_{0}\right)=v_{n}\left\{\frac{\tilde{\nu}}{24}\left(\frac{1}{Q_{L}}+\frac{1}{Q_{S}}\right)+\frac{19}{30}\left(\frac{1}{Q_{L}}-\frac{1}{Q_{S}}\right)\right\}
$$

In performing the integration, we note that the terms involving $G_{L}$ and $G_{S}$ in the integrands of Eq. (43) combine and simplify upon using the heat flux condition (40). Thus the leading order temperature field is continuous across the interface if we choose

$$
\widetilde{\nu}=12\left(Q_{L}-Q_{S}\right)=24\left(\frac{\kappa_{L}-\kappa_{S}}{\kappa_{L}+\kappa_{S}}\right)
$$

in the case $r(\phi)=\phi$, and choose

$$
\widetilde{\nu}=\frac{38}{5}\left(Q_{L}-Q_{S}\right)=\frac{76}{5}\left(\frac{\kappa_{L}-\kappa_{S}}{\kappa_{L}+\kappa_{S}}\right)
$$

in the case $r(\phi)=p(\phi)$. Values of $\widetilde{\nu}$ for other choices for $r(\phi)$ can be calculated in a similar manner to ensure that the interfacial temperature is continuous. Almgren [2] discusses 
strategies for the standard model to ensure that the usual boundary conditions are satisfied with $O\left(\epsilon^{2}\right)$ errors as well.

\subsubsection{The Modified Gibbs-Thomson Equation}

We now investigate the solvability condition (38) to determine the form of the modified Gibbs-Thomson equation in the thin interface limit. Evaluating the integrals in Eq. (38) and using the relation

$$
G_{S}-G_{L}=\frac{v_{n}}{2}\left[\frac{1}{Q_{L}}+\frac{1}{Q_{S}}\right]-\bar{G}\left[\frac{1}{Q_{L}}-\frac{1}{Q_{S}}\right]
$$

where $\bar{G}=\left(Q_{L} G_{L}+Q_{S} G_{S}\right) / 2$ is the (conductivity-weighted) average temperature gradient, gives that the solvability equation can be written in the form

$$
\begin{aligned}
\frac{\lambda}{2}\left(U_{+}^{(1)}+U_{-}^{(1)}\right)= & v_{n}\left\{\left(\frac{3 \lambda \tilde{\nu}}{560}+\frac{\beta}{240}\right)\left[\frac{1}{Q_{L}}-\frac{1}{Q_{S}}\right]+\left(\frac{5 \lambda}{12}-\frac{\tilde{\beta} \widetilde{\nu}}{1120}\right)\left[\frac{1}{Q_{L}}+\frac{1}{Q_{S}}\right]-\frac{\tau}{6}\right\} \\
& -\bar{G}\left\{\frac{5 \lambda}{6}\left[\frac{1}{Q_{L}}-\frac{1}{Q_{S}}\right]+\frac{\widetilde{\beta}}{24}\left[\frac{1}{Q_{L}}+\frac{1}{Q_{S}}\right]\right\}-\frac{\widetilde{\mathcal{H}}}{6}
\end{aligned}
$$

for $r(\phi)=\phi$, and

$$
\begin{aligned}
\frac{\lambda}{2}\left(U_{+}^{(1)}+U_{-}^{(1)}\right)= & v_{n}\left\{\left(\frac{\lambda \tilde{\nu}}{144}+\frac{\tilde{\beta}}{144}\right)\left[\frac{1}{Q_{L}}-\frac{1}{Q_{S}}\right]+\left(\frac{19 \lambda}{60}-\frac{\tilde{\beta} \widetilde{\nu}}{1120}\right)\left[\frac{1}{Q_{L}}+\frac{1}{Q_{S}}\right]-\frac{\tau}{6}\right\} \\
& -\bar{G}\left\{\frac{19 \lambda}{30}\left[\frac{1}{Q_{L}}-\frac{1}{Q_{S}}\right]+\frac{\widetilde{\beta}}{24}\left[\frac{1}{Q_{L}}+\frac{1}{Q_{S}}\right]\right\}-\frac{\widetilde{\mathcal{H}}}{6}
\end{aligned}
$$

for $r(\phi)=p(\phi)$.

The term proportional to $\bar{G}$ is eliminated if we choose

$$
\tilde{\beta}=10 \lambda\left(Q_{L}-Q_{S}\right)=20 \lambda\left(\frac{\kappa_{L}-\kappa_{S}}{\kappa_{L}+\kappa_{S}}\right)
$$


in the case $r(\phi)=\phi$, and

$$
\widetilde{\beta}=\frac{38}{5} \lambda\left(Q_{L}-Q_{S}\right)=\frac{76}{5} \lambda\left(\frac{\kappa_{L}-\kappa_{S}}{\kappa_{L}+\kappa_{S}}\right)
$$

in the case $r(\phi)=p(\phi)$.

Inserting the expressions (52) or (53) for $\widetilde{\beta}$ and (47) or (48) for $\widetilde{\nu}$ into the solvability condition then gives

$$
6 \lambda U^{(1)}=-v_{n}\left\{\tau-\frac{c_{1} \lambda}{Q_{L} Q_{S}}\left[1-c_{2}\left(Q_{L}-Q_{S}\right)^{2}\right]\right\}-\widetilde{\mathcal{H}}
$$

where $c_{1}$ and $c_{2}$ are purely numerical constants whose values are given by $c_{1}=5$ and $c_{2}=269 / 700$ for $r(\phi)=\phi$, and $c_{1}=19 / 5$ and $c_{2}=173 / 525$ for $r(\phi)=p(\phi)$. This expression can be written in terms of dimensional variables as

$$
u=\epsilon U^{(1)}=-V / V_{0}-\ell_{c} H,
$$

where $V_{0}=\mu\left[L_{V} / c_{P}\right]$ and

$$
\frac{1}{\mu}=\frac{T_{M}}{6 \ell M L_{V}}-\frac{c_{1} \ell \bar{\kappa}\left[L_{V} / c_{P}\right]}{6 \kappa_{L} \kappa_{S}}\left[1-4 c_{2}\left(\frac{\kappa_{L}-\kappa_{S}}{\kappa_{L}+\kappa_{S}}\right)^{2}\right] .
$$

Following Karma and Rappel [1], we note that the effects of kinetics can be eliminated $(1 / \mu=0)$ by the choice

$$
\frac{1}{M}=\frac{c_{1} \ell^{2} \bar{\kappa}\left[L_{V}^{2} / c_{P}\right]}{\kappa_{L} \kappa_{S} T_{M}}\left[1-4 c_{2}\left(\frac{\kappa_{L}-\kappa_{S}}{\kappa_{L}+\kappa_{S}}\right)^{2}\right] .
$$

In order that the phase-field equation is well posed we require that $M>0$ and so (57) may be satisfied providing that the ratio $\kappa_{S} / \kappa_{L}$ is not too extreme; specifically

$$
\frac{2 \sqrt{c_{2}}-1}{2 \sqrt{c_{2}}+1}<\frac{\kappa_{S}}{\kappa_{L}}<\frac{2 \sqrt{c_{2}}+1}{2 \sqrt{c_{2}}-1}
$$


which evaluates to $0.107<\kappa_{S} / \kappa_{L}<9.34$ for $r(\phi)=\phi$ and $0.0689<\kappa_{S} / \kappa_{L}<14.5$ for $r(\phi)=p(\phi)$. Both of these criteria are met by most semiconductors and metals as well as succinonitrile.

\section{Discussion}

In this section we address a number of related issues that concern the asymptotic analysis. We first discuss the elimination of anomalous terms by using the generalized phase-field model, followed by an indication of how surface tension anisotropy can be included in the model. The magnitudes of the anomalous terms in the thin interface analysis of the standard phase-field model are then estimated. We conclude the section by comparing the assumed scalings for the classical and thin interface asymptotics.

\subsection{Elimination of Anomalous Terms}

In the isothermal analysis, we find that of the four parameters $\alpha, \beta, \delta$, and $\nu$ that occur in the generalized phase field equations, only two parameters, $\widetilde{\beta}=\beta-\alpha$ and $\widetilde{\nu}=\nu-\delta$, appear as degrees of freedom that are available in the first order analysis to ensure that the interfacial temperature $U^{(1)}$ is continuous and that the anomalous term that is proportional to the temperature gradient in the Gibbs-Thomson equation vanishes. If, instead of assuming the independence of the dimensionless parameters $\alpha, \beta, \delta$, and $\nu$ we consider their definitions in terms of the parameters $K_{E}, K_{S}, W_{E}$ and $W_{S}$ that appear in the internal energy and entropy functions, we find that $\widetilde{\beta}$ and $\widetilde{\nu}$ are actually not independent, but are related, since Eq. (22) implies that

$$
\widetilde{\beta}=\lambda \widetilde{\nu}=-\frac{L_{V}}{36 c_{P} \gamma^{2}}\left(W_{E} K_{S}-W_{S} K_{E}\right) .
$$

Thus, if the parameters in the generalized phase-field equations have values that are derived from an underlying internal energy and entropy functions in a thermodynamically-consistent manner, only a single degree of freedom, namely $W_{E} K_{S}-W_{S} K_{E}$, is available to satisfy the two conditions. However, we note that for the generalized gradient equations, these conditions 
given by (48) and (53) are linearly independent and reduce to

$$
\widetilde{\beta}=\lambda \widetilde{\nu}=\lambda \widetilde{\nu}^{\star},
$$

where

$$
\widetilde{\nu}^{\star}=\frac{76}{5}\left(\frac{\kappa_{L}-\kappa_{S}}{\kappa_{L}+\kappa_{S}}\right) .
$$

Hence, in the fully thermodynamically consistent treatment, represented by the generalized gradient equations, we can choose the four parameters $\alpha, \beta, \delta, \nu$ so that both the temperature at the interface is continuous and the Gibbs-Thomson equation is independent of the interfacial temperature gradients. In addition, for a wide range of values of $\kappa_{S} / \kappa_{L} M$ may be chosen using equation (57) so that kinetic effects are not present, i.e., the dimensional boundary conditions on the value of the interfacial temperature are

$$
T_{S}=T_{L}=T_{M}-\frac{\ell_{c} L_{V}}{c_{P}} \mathcal{H}
$$

where $T_{S}$ and $T_{L}$ are the interfacial temperatures in the solid and liquid respectively. We observe that the choice of $\alpha, \beta, \delta, \nu$ consistent with (60) is not unique. For example, we may choose $\alpha=\delta=0, \beta / \lambda=\nu=\widetilde{\nu}^{\star}$, which corresponds to allowing the bulk free energy double well height $W=W_{E}+T W_{S}$ to depend on $T$ and the gradient energy coefficient $K_{F}=K_{E}+T K_{S}$ to be independent of $T$. Similarly, the choice $\beta=\nu=0, \alpha / \lambda=\delta=-\widetilde{\nu}^{\star}$, corresponds to the opposite situation where $W$ is independent of $T$ and $K_{F}$ is dependent on $T$. This latter choice may be advantageous in numerical computation using the generalized gradient equations since it eliminates the $\phi_{t} \nabla^{2} \phi$ term in the Eq.(20). In the nongradient case it is still possible to eliminate the anomalous terms using the choices for $\widetilde{\beta}$ and $\widetilde{\nu}$ given by equations (53) and (48), respectively; however, in this case the condition between them given by equation (59) will not be satisfied.

The isothermal asymptotics applied to either of the generalized phase-field equations gives interfacial conditions that depend on the forms adopted for the functions $r(\phi), Q(\phi)$ 
and $p(\phi)$. In particular, the results given above for the generalized gradient equations are for the specific choice $p(\phi)=\phi^{2}(3-2 \phi)$ and the form of $Q(\phi)$ given by equation (44). We conjecture that for a more general form of $p(\phi)$ the elimination of the anomalous terms will still require the relationship $\widetilde{\beta}=\lambda \widetilde{\nu}$ to hold but will alter the expression for $\widetilde{\nu}^{\star}$. In contrast, the classical asymptotics gives interfacial conditions on the temperature that are independent of the forms of $r(\phi), Q(\phi)$ and $p(\phi)$.

In the thin interface limit, the sensitivity of the limiting interfacial boundary conditions to the details of the model within the interface can be attributed to the relatively large interfacial thickness allowed by this limit as compared to the classical sharp interface limit. A similar sensitivity was also observed in a different thin interface analysis of solute trapping for a phase field model of a binary alloy [28]. In this situation the dependence on the details of the phase-field model in the interfacial region was apparent in an asymptotic regime where the interface thickness was also comparatively large, in fact comparable to the solute boundary layer thickness.

We note that for both the classical analysis and the thin interface analysis the temperature field near the interface is spatially uniform to leading order in $\epsilon$; in the thin interface analysis $T=T_{M}$ to leading order, and in the classical analysis there are corrections to $T_{M}$ of order unity due to capillary and kinetic terms. The first order correction to the temperature field is non-constant in each analysis, with an $O(\epsilon)$ spatial variation in temperature through the interface.

\subsection{Anisotropic Surface Energy}

The phase field equations (19) and (20) are based on isotropic forms of the gradient energy and gradient entropy represented by the $|\nabla \phi|^{2}$ terms in the functionals (7) and (8). A model with anisotropy can be obtained by replacing $|\nabla \phi|^{2}$ in these expressions by the term $[\Gamma(\nabla \phi)]^{2}$, where $\Gamma(\vec{p})$ is a homogeneous function of degree one $[8,25-27]$. The introduction

of the function $\Gamma(\nabla \phi)$ provides a phase-field model of interfaces whose anisotropic surface free energy is proportional to $\Gamma(\widehat{n})$ in the sharp interface limit, where $\widehat{n}$ is the outward unit 
normal to the interface. For example, the function

$$
\Gamma(\nabla \phi)=|\nabla \phi|\left\{1+\gamma_{4} \cos \left(4 \arctan \left[\phi_{y} / \phi_{x}\right]\right)\right\}
$$

models a two-dimensional interface with a four-fold surface energy proportional to $\Gamma(\widehat{n})=$ $\left(1+\gamma_{4} \cos 4 \theta\right)$, where $\theta$ is the orientation of $\widehat{n}=(\cos \theta, \sin \theta)$. The resulting anisotropic forms of the generalized plase-field equations are obtained by the substitution

$$
\nabla^{2} \phi \rightarrow \nabla \cdot[\Gamma \vec{\xi}]
$$

in equations (19) and (20), where $\vec{\xi}(\nabla \phi)$ is the Cahn-Hoffman $\xi$-vector [30] with components $\xi_{j}(\vec{p})=\partial \Gamma(\vec{p}) / \partial p_{j}$. The sharp interface analysis of the anisotropic equations parallels the treatment in Ref. [25]; for brevity, we omit the details. The final result is that the mean curvature term $\mathcal{H}$ in the Gibbs-Thomson equation (55) is replaced by the weighted mean curvature term $\nabla_{S} \cdot \vec{\xi}$ involving the surface divergence of the $\xi$-vector, which is the appropriate generalization of the Gibbs-Thomson equation for the anisotropic case [31,32].

\subsection{Magnitudes of Anomalous Terms in the Thin Interface Limit}

In Section 3.1 we sketch the classical analysis for the standard model. It is straightforward to perform the classical analysis for the generalized model as well. One finds that the leading order temperature is continuous at the interface and satisfies a non-linear version of the usual (modified) Gibbs-Thomson equation. The conventional leading-order balance of heat flux at the interface is also obtained, but with a discontinuity in the first-order temperature field across the interface. It is this observation that suggests the use of the generalized phase-field model in the thin interface limit in this paper to eliminate the temperature discontinuity that arises for unequal thermal conductivities.

The thin interface limit of the conventional phase-field model also leads to a jump in temperature at the interface given by Eq. (45) or (46) with $\tilde{\nu}=0$. Considering as an example the case $r(\phi)=\phi$, the dimensional form of the leading order temperature jump can 
be written as

$$
T_{L}-T_{S}=\left[L_{V} / c_{P}\right]\left(1-\frac{k_{L}}{k_{S}}\right)\left[\frac{\ell V}{\kappa_{L}}\right]
$$

which shows that the temperature jump vanishes if the thermal properties of liquid and solid are equal. The jump in temperature is a non-equilibrium effect that resembles in some ways the "solute trapping" effect that is observed for rapid solidification of a binary alloy [33]. Solute trapping involves non-equilibrium partitioning in the solute at the solid-liquid interface due to a jump in chemical potential across the interface. The jump in potential becomes significant at velocities $V \sim V_{D}$, where the characteristic velocity $V_{D}$ is given by the ratio of an interfacial solute diffusivity $D_{I}$ to the interface width $\ell$. In a similar way, the temperature jump in (65) is proportional to $V / V_{T}$, where $V_{T}=\kappa_{L} / \ell$ is a characteristic thermal velocity. In phase-field models of solute trapping, $V_{D}$ is found to be sensitive to the assumed form of the variation of the solute diffusivity through the interface [28], just as the numerical factors in Eqs. (45) and (46) depend on the assumed form for $r(\phi)$. The sensitivity of the limiting interfacial boundary conditions to the details of the model within the interface in the thin interface limit can be attributed to the relatively large interfacial thickness allowed by this limit, as compared to the classical sharp interface limit. For solute trapping the appropriate limit also involves an asymptotic regime where the interface thickness is relatively large, in fact comparable to the solute boundary layer thickness. For solute trapping in metallic alloys a typical value of $V_{D}$ is on the order of $1 \mathrm{~m} / \mathrm{s}$; since the thermal diffusivities are usually orders of magnitude larger than typical solute diffusivities, the temperature jump is a much smaller effect. Using values for lead as an example with $k_{S} / k_{L} \approx 2$, a nominal interface thickness of $10^{-7} \mathrm{~cm}$ leads to a temperature jump of about $10^{-2} \mathrm{~K}$ at a velocity of $1 \mathrm{~m} / \mathrm{s}$.

The thin interface limit also results in an anomalous term in the modified Gibbs-Thomson equation, which for the case $r(\phi)=\phi$ can be written in the dimensional form

$$
\frac{\left(T_{L}+T_{S}\right)}{2}=T_{M}-\left[L_{V} / c_{P}\right] \ell_{c} \mathcal{H}-V / \mu-\frac{5}{12} \ell\left[k_{L} \frac{\partial T_{L}}{\partial n}+k_{S} \frac{\partial T_{S}}{\partial n}\right]\left(\frac{1}{k_{L}}-\frac{1}{k_{S}}\right),
$$


where the last term in this expression depends on the conductivity-weighted temperature gradient at the interface. This term is a very small correction for metallic systems under most conditions; for example, using values for lead with a mean temperature gradient of 100 $\mathrm{K} / \mathrm{cm}$ and $\ell=10^{-7} \mathrm{~cm}$ gives a correction of roughly $10^{-5} \mathrm{~K}$.

\subsection{Scalings of Surface Energy and Kinetics}

We now return to the question of the assumed scaling of capillary and kinetics effects in the classical and thin interface asymptotics for the standard equations. We couch the discussion in terms of the dimensionless parameters $\epsilon=\ell / R, \lambda=\ell /\left(6 \ell_{c}\right)$, and $\tau$, where we recall that $\ell$ is the interface width, $R$ is a macroscopic length scale, $\ell_{c}=T_{M} \gamma /\left[L_{V}^{2} / c_{P}\right]$ is the capillary length, and $\tau$ is a dimensionless form of the mobility coefficient $M$ [cf. Eq. (21)] that is assumed to be of order unity in both analyses. The two asymptotic limits correspond to $\epsilon \rightarrow 0$ with either $\lambda=O(\epsilon)$ (the classical analysis) or $\lambda=O(1)$ (the thin interface analysis). The gradient energy coefficient $K$, double well height $W$, and mobility coefficient $M$ can be written in the form

$$
K=6\left(\frac{L^{2} R^{2}}{c_{P} T_{M}}\right) \frac{\epsilon^{2}}{\lambda}, \quad W=6\left(\frac{L^{2}}{c_{P} T_{M}}\right) \frac{1}{\lambda}, \quad M=\left(\frac{\bar{\kappa} T_{M} c_{P}}{\tau L_{V}^{2} R^{2}}\right) \frac{\lambda}{\epsilon^{2}} .
$$

In the classical analysis the surface energy and kinetic coefficients have the form

$$
\gamma=\left(\frac{L^{2} R}{c_{P} T_{M}}\right) \frac{\epsilon}{\lambda}, \quad \frac{1}{\mu}=\left(\frac{\tau L_{V} R}{6 c_{P} \bar{\kappa}}\right) \frac{\epsilon}{\lambda} .
$$

In the thin interface analysis, $\gamma$ also has this form, but $1 / \mu$ has an additional term as given by Eq. (56). In the classical analysis with $\lambda=O(\epsilon), \gamma$ and $1 / \mu$ scale as $O(1)$ quantities with respect to $\epsilon$, the gradient energy coefficient is $O(\epsilon)$, the double well height is $O(1 / \epsilon)$, and the mobility is $O(1 / \epsilon)$. In the thin interface limit with $\lambda=O(1), \gamma$ tends to zero as $O(\epsilon)$, the gradient energy coefficient tends to zero as $O\left(\epsilon^{2}\right)$, and the double well height scales as an $O(1)$ quantity. The interpretation of the kinetic coefficient in this limit is more involved, since one can arrange that $1 / \mu=0$ by an appropriate choice of $M$ as given by Eq. (57). 
With this choice, $M$ scales as $O\left(1 / \epsilon^{2}\right)$ which is consistent with the scaling given by Eq. (67) with $\lambda=O(1)$. This shows that, in the thin interface limit, the effects of capillarity and kinetics are assumed to be small corrections in the analysis, as noted in the introduction.

In the context of numerical calculations using a phase-field formulation, the two asymptotic analyses suggest different interpretations of a given computation, and different strategies for the appropriate selection of parameters for a sequence of calculations that are intended to reproduce a sharp-interface model. With the classical analysis, to converge to a sharp interface result one should in principle perform a sequence of calculations with $\ell$ tending to zero for fixed values of $\gamma$ and $1 / \mu$, so that $\epsilon$ and $\lambda$ both tend to zero. By contrast, for the thin interface analysis, the surface energy and kinetic effects vanish as $\ell$ tends to zero, and in principle a single calculation with a fixed (small) value of $\epsilon$ is performed, with appropriate values prescribed for $K, W$, and $M$ in order to give the desired values of $\gamma$ and $1 / \mu$ consistent with the chosen value of $\epsilon$. Performing a convergence study with $\epsilon$ tending to zero in the thin interface limit requires constant reduction of $\lambda$ to preserve given values of surface energy and kinetics, causing the procedure to revert to the classical analysis in the limit of small $\epsilon$. On the other hand, lower bounds on the feasible size of $\epsilon$ are set by computational constraints in practise, so convergence studies of this nature have limited applicability.

\section{Conclusions}

The thin interface asymptotics of Karma and Rappel applied to the standard phase-field equations for a pure material with unequal thermal conductivities generate anomalous terms involving a temperature jump across the interface and a contribution from the mean temperature gradient in the modified Gibbs-Thomson equation [1,2]. The temperature discontinuity can be interpreted as a thermal trapping effect analogous to the solute trapping that occurs during the rapid solidification of a binary alloy. For metallic systems under typical growth conditions both anomalous terms are very small, and can be eliminated by employing a generalized phase-field model that is based on entropy and internal energy gradient energy 
functionals that include double wells in both the internal energy and entropy densities. The flexibility of the thin interface analysis in eliminating kinetic effects carries over to the case of unequal thermal conductivities as well, as also noted by Almgren in his isothermal analysis of this limit. In our approach kinetic effects may be eliminated for conductivity ratios of up to about 10:1. The use of thin interface asymptotics to eliminate kinetic effects in binary alloy systems where diffusivities may differ by several orders of magnitude is an area of active research, where the use of generalized energy-entropy models may play a useful role.

\section{Acknowledgments}

The authors are grateful for helpful discussions with W.J. Boettinger, S.R. Coriell, and R.F. Sekerka. This work was conducted with the support of the Microgravity Research Division of NASA.

\section{References}

[1] A. Karma and W.-J. Rappel, Phys. Rev. E 53 (1996) R3017; Phys. Rev. E 57 (1998) 4342 .

[2] R. F. Almgren, Second-order phase-field asymptotics for unequal conductivities, to appear in SIAM J. Appl. Math.

[3] G. Caginalp, Phys. Rev. A 39 (1989) 5887.

[4] M.E. Glicksman and S.P. Marsh, The Dendrite, in Handbook of Crystal Growth, Vol. 1b, ed. D. T. J. Hurle, (Elsevier, Amsterdam, 1993), p. 1075.

[5] G. Caginalp, Arch. Rat. Mech. Anal. 92 (1986) 205.

[6] P.C. Fife, Dynamics of Internal Layers and Diffusive Interfaces, CBMS-NSF Regional Conference Series in Applied Mathematics, Vol. 53 (SIAM, Philadelphia, 1988).

[7] G.B. McFadden, A.A. Wheeler, R.J. Braun, S.R. Coriell, and R.F. Sekerka, Phys. Rev. E 48 (1993) 2016.

[8] E. Fried and M.E. Gurtin, Physica D 72 (1994) 287. 
[9] P. C. Fife and O. Penrose, Electronic J. Diff. equations. 1 (1995) 1.

[10] J.W. Cahn, P. Fife, and O. Penrose, Acta Mater. 45 (1997) 4397.

[11] B.I. Halperin, P.C. Hohenberg, and S.-K. Ma, Phys. Rev. B 10 (1974) 139.

[12] J. S. Langer. Unpublished notes.

[13] J.B. Collins and H. Levine, Phys. Rev. B 31 (1985) 6119.

[14] A.R. Umantsev and A.L. Roitburd, Sov. Phys. Solid State 30 (1985) 651.

[15] J. S. Langer, in Directions in Condensed Matter Physics, ed. G Grinstein, G Mazenko, 165-86. Philadelphia: World Scientific (1986).

[16] G. Caginalp, in Applications of Field Theory to Statistical Mechanics, edited by L. Garrido, Lecture Notes in Physics Vol. 216 (Springer-Verlag, Berlin, 1986) p. 216.

[17] O. Penrose and P.C. Fife, Physica D 43 (1990) 44.

[18] S.-L. Wang, R.F. Sekerka, A.A. Wheeler, B.T. Murray, S.R. Coriell, R.J. Braun, and G.B. McFadden, Physica D 69 (1993) 189.

[19] A. P. Umantsev, J. Chem. Phys. 96 (1992) 605.

[20] H.W. Alt and I. Pawlow, Physica D 59 (1992) 389.

[21] A.A. Wheeler, G.B. McFadden, and W.J. Boettinger, Proc. Roy. Soc. Lond. A 452 (1996) 495.

[22] E. Fried and M.E. Gurtin, Physica D 91 (1996) 143.

[23] C. Charach and P. C. Fife, On thermodynamically consistent schemes for phase-field equations. To appear in Open Systems and Information Dynamics.

[24] D.M. Anderson, G.B. McFadden, and A.A. Wheeler, Physica D (1999), in press.

[25] A.A. Wheeler, G.B. McFadden, Euro. J. Appl. Math. 7 (1996) 367.

[26] A.A. Wheeler, G.B. McFadden, Proc. Roy. Soc. Lond. A 453 (1997) 1611.

[27] E. Fried and M.E. Gurtin, Physica D 68 (1993) 326.

[28] N.A. Ahmad, A.A. Wheeler, W.J. Boettinger, and G.B. McFadden, Phys. Rev. E 58 (1998) 3436.

[29] A. Umantsev, Acta Mater. 46 (1998) 4935.

[30] J.W. Cahn D.W. Hoffman, Acta Metall. 22 (1974) 1205. 
[31] J. Taylor, Acta Metall. Mater. 40 (1992) 1475.

[32] J. Taylor and J.W. Cahn, J. Stat. Phys. 77 (1994) 183.

[33] M.J. Aziz, Metall. Mater. Trans. A 27 (1996) 671. 

